\title{
Crowd Salience Heightens Tolerance to Healthy Facial Features
}

\author{
Mitch Brown ${ }^{1}$ (I) Ryan E. Tracy ${ }^{2}$ Steven G. Young ${ }^{2,3}$ - Donald F. Sacco ${ }^{4}$
}

Received: 23 April 2021 / Revised: 14 September 2021 / Accepted: 15 September 2021 /

Published online: 21 September 2021

() The Author(s), under exclusive licence to Springer Nature Switzerland AG 2021

\begin{abstract}
Objective Recent findings suggest crowd salience heightens pathogen-avoidant motives, serving to reduce individuals' infection risk through interpersonal contact. Such experiences may similarly facilitate the identification, and avoidance, of diseased conspecifics. The current experiment sought to replicate and extend previous crowding research.
\end{abstract}

Methods In this experiment, we primed participants at two universities with either a crowding or control experience before having them evaluate faces manipulated to appear healthy or diseased by indicating the degree to which they would want to interact with them.

Results Crowding-primed participants reported a more heightened preferences for healthy faces than control-primed participants. Additionally, crowd salience reduced aversion toward healthy faces but did not heighten aversion to diseased faces.

Conclusion Results suggest crowding appears to heighten tolerance for health cues given the heightened proximal threat of infections through interpersonal contact within crowded environments. Conversely, this work extends previous findings by indicating this preference is not rooted in an aversion to cues of poor health. We frame findings from a threat management perspective in understanding how crowding fosters sensitivity toward pathogenic threats.

Keywords Crowding $\cdot$ Disease $\cdot$ Face perception $\cdot$ Avoidance $\cdot$ Approach

Mitch Brown

mb103@uark.edu

1 Department of Psychological Science, University of Arkansas, Fayetteville, AR 72701, USA

2 Graduate Center at CUNY, New York, NY, USA

3 CUNY Baruch College, New York, NY, USA

4 The University of Southern Mississippi, Hattiesburg, MS, USA 


\section{Introduction}

The transmission of communicable disease has become a pervasive threat as human populations grow. Densely populated ecologies are prone to infectious disease through increased interpersonal contact (Jones et al., 2008; Rocklöv \& Sjödin, 2020). Increasing urbanization has led to a greater likelihood of individuals residing in densely populated areas (Bloom et al., 2008). This urbanization naturally increases experiences of crowding that correspondingly increase risk of pathogenic illness exposure. This risk became particularly salient during the COVID-19 pandemic, which saw the employment of extensive social distancing guidelines that reduced the requisite contact for disease spread and a considerable migration from individuals away from urban environments (Gagnon et al., 2020; Whitaker, 2021). The resulting vigilance toward pathogenic threat from interpersonal contact would similarly elicit motivations and behaviors to ensure individuals the opportunity to maintain access to benefits of group living while minimizing risk of disease spread.

In addition to disease salience eliciting perceptions of crowded spaces as more threatening (Wang \& Ackerman, 2019), crowd salience elicits perceptions of oneself as vulnerable to disease and motivates interpersonal reticence to reduce the fitness costs of infection (Brown \& Sacco, 2019; Maeng, Tanner, \& Soman, 2013; Sng et al., 2017). This caution may further elicit consideration of which individuals optimize group living in densely populated environments. It could prove advantageous to identify group members whose appearance suggests reduced risk of disease transmission through healthy facial features. Crowd salience could heighten this sensitivity toward these features given the inevitability of interpersonal contact that would warrant identification of likely threats preemptively to prevent contact with diseased conspecifics (Neuberg, Kenrick, \& Schaller, 2011). This study sought to identify how crowd salience activates pathogen-avoidant motives as indexed by subsequent interpersonal preferences based on virulence cues.

\section{Crowding and Threat Management}

Despite myriad benefits of group living, human sociality remains bounded, and oversaturation is possible. One route through which the oversaturation could emerge is the feeling of crowding. Crowding is the psychological discomfort from being in environments in which there are more people occupying a given space than can be comfortably accommodated (Cain \& LeDoux, 2008). Crowding creates a unique experience wherein the risks of exposure to (many) other people far outweigh any benefits. Perceptions of oneself as feeling crowded subsequently elicits feelings of constriction (Gochman \& Keating, 1980; Stockdale, 1978). Such feelings foster motivations for social withdrawal (Baum \& Valins, 1979), heightened anxiety (Schaeffer \& Patterson, 1980), and sympathetic nervous system responses (Aiello et al., 1977; Kennedy et al., 2009).

Subsumed within this social withdrawal are myriad cautious responses to minimize safety and health threats to posed by these environments. Individuals in densely populated ecologies typically express greater disinterest in promiscuous 
mating strategies, a behavioral repertoire that is frequently associated with greater disease transmission (Sng et al., 2017). Recent findings suggest that population density is further associated with reduced fertility rates across 174 nations, a response that could reflect a reduction of potential disease hosts in an already saturated ecology (Rotella et al., 2020). Temporally activating concerns of a crowd further leads to greater interest in consuming various emergency materials serving to reduce risk of illness and injury (Maeng et al., 2013). These findings indicate crowd salience downregulates interest in behaviors that could increase the likelihood of incurring health costs, suggesting their overall salience could heighten aversion to threats beyond those related to physical safety.

\section{Behavioral Components of the Immune System and Social Behavior}

To combat pathogenic threats, organisms possess a sophisticated immunological response system comprised of innate (e.g., cellular barriers) and adaptive subsystems (antigenic responses) that provide a multilayered defense against disease vectors (Litman, Cannon, \& Dishaw, 2005; Pancer \& Cooper, 2006). However, many of their more salient responses toward infections remain metabolically costly (e.g., white blood cells, fevers), with substantial caloric expenditures (i.e., 13\% increase in metabolic activity) to raise one's body temperature by $1{ }^{\circ} \mathrm{C}$ (Kluger, 1991). These immunological responses could thus divert resources away from physiological systems involved in other fitness-enhancing behaviors (e.g., eating, mating). As a complement to these responses, research suggests organisms evolved a concomitant behavioral component of the immune system involved in the identification, and avoidance, of disease vectors preemptively to reduce the likelihood of contracting infections through physical contact to reduce the necessity of relying entirely on physiological responses (Murray \& Schaller, 2016). Behavioral responses to disease cues are common in various species, including perceptual acuity toward diseased conspecifics in rodents (Kavaliers, Choleris, \& Pfaff, 2005), ostracism of infected group members among chimpanzees (Goodall, 1986), and proactive lining of nests with antibiotic resin in ants (Chapuisat et al., 2007).

A growing preponderance of evidence has emerged demonstrating similar responses among humans. Responses include affective disgust toward food with a heightened likelihood of parasite contamination (Al-Shawaf et al., 2015) and specific behaviors designed to remove ectoparasites in the presence of social cues to disease (e.g., contagious itching; Kupfer \& Fessler, 2018). Because of the considerable risk of disease transmission through interpersonal contact (Jones et al., 2008), various interpersonal behaviors have been implicated as responses to this possibility across cultures. Geopolitical regions experiencing greater prevalence of infectious disease frequently see the emergence of cultures that valuate interpersonal reticence and restricted sexual strategies (Schaller \& Murray, 2008). These restrictive behaviors further include adoption of collectivistic values in regions with heightened pathogen loads (Fincher et al., 2008), greater valuation of conformity within the corresponding countries (Murray, Trudeau, \& Schaller, 2011; Wu \& Chang, 2012), and interest in authoritarian governance (Murray, Schaller, \& Suedfeld, 2013). Both chronic and acute concerns of disease further 
foster interpersonal reticence (Brown \& Sacco, 2016; Mortensen et al., 2010; Tybur et al., 2020), aversion to physical contact with others (Brown \& Sacco, 2020; Brown, Young, \& Sacco, 2021; Makhanova \& Shepherd, 2020; Sawada, Auger, \& Lydon, 2018), and downregulated affiliative motives to reduce the physical contact necessary for disease transmission (Sacco, Young, \& Hugenberg, 2014).

Aversive responses to pathogenic threats include the identification of potential disease risk among conspecifics, leading to greater aversion toward those possessing putative cues toward disease. These aversive responses operate within an overgeneralization principle, wherein humans perceive an overabundance of social stimuli as infectious to minimize costly failures in classifying actual threats (Haselton \& Nettle, 2006). Liberal identification criteria foster withdrawal from those whose appearance heuristically connotes disease threat, even if such appearance is not indicative of an actual infection risk (e.g., obesity; Miller \& Maner, 2012). Such responses include vigilance toward disfigurements (Ackerman et al., 2009), aversion to non-normative and unfamiliar conspecifics (Murray \& Schaller, 2012; Peng, Chang, \& Zhou, 2013), and a heightened desire to penalize others (Brown et al., 2017).

\section{Interplays Between Disease Avoidance and Crowds}

Despite the risk of infectious disease in group living, humans are nonetheless willing to incur the potential costs provided they do not exceed the benefits afforded through group living. Exclusionary experiences specifically reduce concerns about contracting infections and heighten individuals' desire to affiliate with those who present increased risk of disease transmission through extensive interpersonal contact (i.e., extraverts; Brown et al., 2019a, 2019b; Sacco et al., 2014). However, the potential benefits of group living remain bounded as individuals could experience an oversaturation in interpersonal contact and begin to recognize the costs as exceeding any potential benefits (Tybur \& Lieberman, 2016).

Densely populated ecologies experience increased likelihood of disease transmission (Hoang et al., 2019; Salathé et al., 2010), which could result in perceptions of socializing in crowds as being higher in costs (i.e., disease transmission) than benefits (i.e., affiliation opportunities). Disease salience amplifies perceptions of crowds as disease vectors (Wang \& Ackerman, 2019), amplifying desires to reduce contact with disease cues. Given this demonstrable interplay between interpersonal contact and disease transmission, recent research has provided further evidence for an implicit understanding of crowds as pathogenically threatening. After rendering a crowding experience salient, individuals reported heightened vigilance toward pathogenic threats (Brown $\&$ Sacco, 2019). This salience further shifted interpersonal preferences by heightening preferences for facial symmetry, a feature associated with reduced pathogen load (Thornhill \& Gangestad, 2006).

\section{Current Research}

This study sought to replicate and extend previous findings demonstrating how crowd salience heightens aversion to disease cues. Crowding indeed heightens 
preferences for facial features putatively associated with health, particularly through facial symmetry (Brown \& Sacco, 2019). However, this preference for symmetry leaves it unclear whether this preference is rooted in an interest in healthy conspecifics or an aversion to those who appear diseased (see Zebrowitz \& Rhodes, 2004), given the specific signal value of symmetry and nature of the preference task in previous work. To reconcile this ambiguity, we specifically considered facial features that would be specifically diagnostic of infection risk instead of reduced pathogen load like with symmetry. Participants were specifically tasked with evaluating target individuals exhibiting skin lesions that are heuristically associated with poor health and foster aversive behaviors among those motivated to avoid disease (van Leeuwen \& Petersen, 2018). This evaluative task further afforded us the opportunity to consider both interest and aversion to associating with targets as a function of their health status directly.

This methodological decision to focus on perceptions of disease cues further afforded us the opportunity to have a comparison with faces that appeared relatively healthy from which we could consider complementary hypotheses related to individuals' motivation to avoid disease. Specifically, we could assess whether evaluations of targets could reflect a preference for healthy facial features in an ostensibly pathogenic environment (Ainsworth \& Maner, 2019; Young, Sacco, \& Hugenberg, 2011), or an aversion to facial features connoting poor health (e.g., Ackerman et al., 2009). If crowding-induced preferences for health cues are rooted in preferences to associate with healthy conspecifics, crowd salience should upregulate interest in healthy conspecifics. Conversely, if this preference is rooted in responses to avoid diseased conspecifics, crowd salience should heighten aversion to disease cues. Data and materials are available through the following link: https://osf.io/n8u6m/.

\section{Methods}

\section{Participants}

We recruited 239 participants from two universities for course credit. One was a public university in a Southern U.S. state $(n=144)$ and the other a private university in a Northeastern U.S. state $(n=95)$ from February to May 2020. No data were excluded from final analyses (190 women, $49 \mathrm{men} ; M_{\text {Age }}=20.43, S D=3.05 ; 52 \%$ White). We collected data at both sites in the service of increasing statistical power in an online study due to constraints imposed by the COVID-19 pandemic that limited data collection opportunities and necessitated use of two sites. We had no a priori predictions for potential differences between universities and did not analyze data until we finished data collection; our stop rule was to collect as many participants as possible until the end of the semester. Sensitivity analyses indicated adequate power for small effects to test for the basic predicted interactive effects for a 2 (Condition: Crowding vs. Control) $\times 2$ (Target Status: Diseased vs. Healthy) mixed experimental design $(f=0.09,1-\beta=0.80)$. 


\section{Materials and Procedure}

\section{Crowd Salience}

Participants were primed by envisioning themselves in one of two public spaces as means to render salient the feelings of being in crowd (Maeng et al., 2013). One space was a large mass of people in a crowded environment $(n=121)$, and the other a few individuals spread far apart as a control condition $(n=118)$. Participants described their hypothetical feelings in either image for a 5-min writing task in a paradigm that has previously demonstrated efficacy in eliciting the salience of a crowd while also being distinct from other negative motivational states (e.g., loneliness, hunger; Brown \& Sacco, 2019). ${ }^{1}$ Participants responded to a single-item general affect item on a 7-point scale $(-3=$ Extremely Negative $;+3=$ Extremely Positive), and 6-item manipulation check assessing feelings of crowding (1=Not at All; $7=$ Very Much; $\alpha=0.95)$.

\section{Target Faces}

Participants evaluated four Caucasian target men, manipulated to present physical cues connoting either health or disease (van Leeuwen \& Petersen, 2018). We presented two unique identities whose faces appeared healthy and two unique identities altered to present physical cues indicating infectious disease. Two versions of each face existed; we counterbalanced which version of was presented to ensure participants evaluated two faces for each category to prevent stimulus effects that were further presented in a randomized order.

Accompanying each target were two questions assessing comfort with proximity to each target in general affiliative domains on 11-point scales $(-5=$ Very Uncomfortable; $0=$ Neutral $; 5=$ Very Comfortable). We aggregated scores for both items in each target category separately and found items for both healthy and diseased faces highly correlated $(r s>0.81, p s<0.001)$. These high correlations prompted us to consider these variables as a singular item for both categories.

Consenting participants were randomly assigned to one of the two priming conditions and responded to the manipulation check. Participants then evaluated target faces before providing demographics and debriefing. ${ }^{2}$

\footnotetext{
1 A supplemental pilot study ( $N=175$ undergraduates) was conducted to demonstrate continuing evidence of the experimental manipulation being capable of activating disease concern, given the relative difficulty in identifying acute changes in pathogen avoidance motives (e.g., Brown \& Sacco, 2019; Makhanova, Plant, \& Maner, 2021). We used a single-item question directly assessing how concerned one felt about disease following the priming $(1=$ Not at All Concerned; $7=$ Very Concerned $)$. Crowdingprimed participants reported greater pathogenic concern $(M=4.78, S D=2.17)$ than did control-primed participants $(M=2.00, S D=1.58), t(159.17)=9.69, p<0.001, d=1.46$.

2 We additionally assessed individual differences in perceived vulnerability to disease (Duncan, Schaller, \& Park, 2009), though this measure elicited no interactive effects with the relevant study variables. Analyses considering this measure are available in the OSF link.
} 


\section{Results}

\section{Preliminary Analyses}

An independent samples $t$-test indicated crowding-primed participants reported feeling more crowded $(M=5.07, S D=1.42)$ than control-primed participants $(M=2.03$, $S D=1.09), t(224.71)=18.48, p<0.001, d=2.38$. Crowding-primed participants additionally reported more negative affect $(M=-0.19, S D=0.67)$ than controlprimed participants $(M=0.67, S D=1.21), t(237)=-4.85, p<0.001, d=-0.62$. Crowding-primed participants' greater negative affect prompted us to consider whether results were influenced by feelings of crowding or affect. This possibility led us to include affect as a covariate in this model to determine the unique role of crowd salience independent of the changes in affect that could influence interpersonal decision-making (see Brown \& Sacco, 2020). ${ }^{3}$

\section{Primary Analyses}

We entered participants' collapsed affiliation responses into a 2 (Condition: Crowding $=1$, Control $=-1) \times 2$ (Region: South $=1$, North $=-1) \times 2$ (Target Status: Diseased $=1$, Healthy $=-1$ ) linear mixed effects model that included random intercepts of participants and target stimuli, a correlated random slope of Target Status for participants and an uncorrelated random slope of Target Status for stimuli, and nested participants in Region. Two-level factors were contrast-coded as is recommended when using such models (see Judd, McClelland, \& Ryan, 2017). ${ }^{4}$ Our decision to collect data at two different author-affiliated universities in separate U.S. regions led us to use the region as a between-subjects factor to identify possible differences in responses on an exploratory basis. Overall model fit indices indicate that a moderate amount of variance is accounted for by both the fixed and random effects, $R_{\text {marginal }}^{2}=.193, I C C_{\text {conditional }}=.629 .{ }^{5}$ Data are summarized in Fig. 1.

A Target Status main effect emerged indicating that participants showed less aversion toward contact with a healthy target $(M=-0.48, S D=2.34)$ than toward a diseased target $(M=-2.36, S D=2.41), b=-.91, S E=.09, t(3.60)=-8.43$, $p=.002, \beta=-.37,95 \% \mathrm{CI}_{\beta}[-.45,-.29], \eta_{\mathrm{p}}{ }^{2}=.95$. A main effect of Region also emerged indicating Southern participants were less likely to have contact with

\footnotetext{
3 Not including affect in the model as a covariate yielded similar findings for the interactions reported herein, although the non-significant Condition $\times$ Region interaction became conventionally significant with its inclusion in the model.

4 A complementary, and similarly dimensioned mixed-model ANOVA yielded parallel results to the effects reported herein.

5 We ran several models with simpler random effects structures to determine which model provided the best fit. These models ranged from the simplest random effects structure (only random intercepts for participants and stimuli) to the most complex, reported here. A model comparison test found that the most complex model provided a significantly improved fit over the simpler model, $\chi^{2}(2)=118.39, p<.001$.
} 


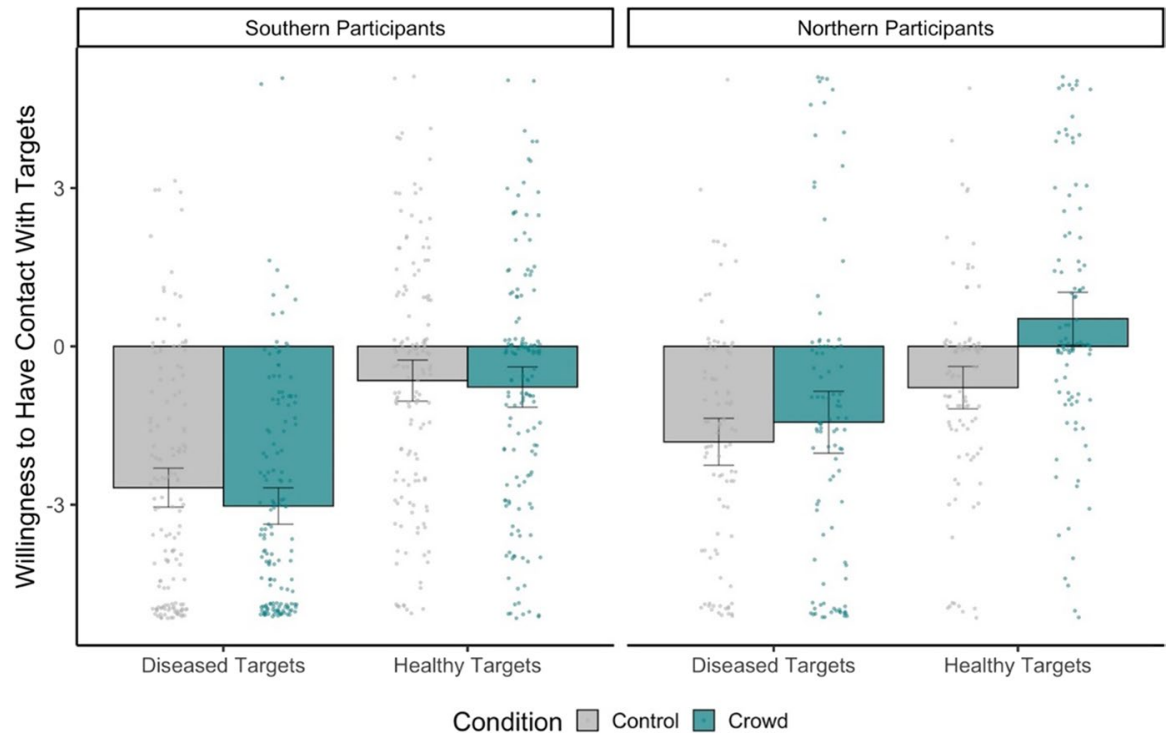

Fig. 1 Average willingness to have contact with diseased versus healthy targets for Southern (left panel) and Northern (right panel) participants, separated by crowded versus control condition. Error bars represent $95 \%$ CIs of the means

any individual $(M=-1.78, S D=2.50)$ than Northern participants $(M=-.87$, $S D=2.55), b=-.47, S E=.13, t(234.03)=-3.64, p<.001, \beta=-.18,95 \% \mathrm{CI}_{\beta}$ $[-.28,-.08], \eta_{\mathrm{p}}{ }^{2}=.05$. The Condition main effect was additionally not significant, $b=.23, S E=.13, t(234.32)=1.69, p=.09, \beta=.07,95 \% \mathrm{CI}_{\beta}[-.03, .17]$, $\eta_{\mathrm{p}}{ }^{2}=.01$. A main effect of Affect as the covariate was significant, $b=.19, S E=.09$, $t(233.99)=2.06, p=.04, \beta=.11,95 \% \mathrm{CI}_{\beta}[.01, .21], \eta_{\mathrm{p}}{ }^{2}=.02$.

\section{Predicted Interaction}

These main effects were initially qualified by a significant Target Status $\times$ Condition interaction, $b=-.15, S E=.05, t(234.99)=-2.24, p=.02, \beta=-.05,95 \% \mathrm{CI}_{\beta}$ $[-.10, .00], \eta_{\mathrm{p}}{ }^{2}=.02$. We decomposed this interaction, while including the Affect covariate in each model, to explore the effect of Target Status for each Condition separately. For control-primed participants, we found a significant effect of Target Status, such that participants evinced a much lower desire to contact a diseased $(M=-2.33, S D=2.24)$ versus a healthy target $(M=-.70, S D=2.20), b=-.81$, $S E=.11, t(7.29)=-7.29, p<.001, \beta=-.35,95 \% \mathrm{CI}_{\beta}[-.44,-.25], \eta_{\mathrm{p}}{ }^{2}=.98$. For crowding-primed participants, there was again a significant effect of Target Status, wherein participants again showed less desire to contact a diseased $(M=-2.39$, $S D=2.57)$ than a healthy target $(M=-.26, S D=2.46), b=-1.07, S E=.13$, $t(4.47)=-8.16, p<.001, \beta=-.39,95 \% \mathrm{CI}_{\beta}[-.49,-.30], \eta_{\mathrm{p}}{ }^{2}=.88$. Neither model yielded a significant effect of the Affect covariate ( $p s \geq .11)$. As evidenced by the standardized beta values, the aversion to diseased faces was magnitudinally larger in the crowding condition. 
We then conducted subsidiary analyses using one-sample $t$-tests to determine where participants' aversion to diseased and healthy faces were categorical aversions. We tested crowded and control participants' contact scores separately against the scalar midpoint of 0 (i.e., neither preference nor aversion) for healthy and diseased targets (Brown, Sacco, \& Medlin, 2019c). Crowding-primed participants showed neither an aversion nor a preference in their contact ratings toward healthy targets, $t(241)=-1.62, p=.11, d=-.15$. However, these same participants demonstrated a strong aversion to diseased targets, $t(241)=-14.50, p<.001, d=-1.32$. Control-primed participants conversely showed an aversion to healthy targets, $t(235)=-4.90, p<.001, d=-.46$, while showing a stronger aversion to diseased targets, $t(235)=-15.98, p<.001, d=-1.47$. Taken together, these findings indicate that crowd salience fosters tolerance toward physical features diagnostic of health rather than aversion to those appearing diseased.

\section{Exploratory Interactions}

Effects were further qualified by an unexpected, albeit significant, Target Status $\times$ Region interaction, $b=-.16, S E=.06, t(234.99)=-2.49, p=.01, \beta=-.06$, $95 \% \mathrm{CI}_{\beta}[-.11,-.01], \eta_{\mathrm{p}}{ }^{2}=.03$. We decomposed this interaction by exploring the effect of target status separately for Northern and Southern participants. For Northern participants, the effect of Target Status was significant, wherein they demonstrated more aversion toward a diseased target $(M=-1.62, S D=2.56)$ than a healthy target $(M=-.12, S D=2.31), b=-.75, S E=.10, t(10.65)=-7.52, p<.001$, $\beta=-.29,95 \% \mathrm{CI}_{\beta}[-.37,-.22], \eta_{\mathrm{p}}{ }^{2}=.84$. For Southern participants, we also found a significant effect of Target Status, wherein participants again were less willing to have contact with diseased targets $(M=-2.85, S D=2.18)$ than healthy targets $(M=-.71, S D=2.34) b=-1.07, S E=.13, t(3.85)=-7.76, p=.002, \beta=-.43$, $95 \% \mathrm{CI}_{\beta}[-.54,-.32], \eta_{\mathrm{p}}{ }^{2}=.94$. This latter comparison was at a larger magnitude.

The Condition $\times$ Source interaction was not conventionally significant, $b=-.24$, $S E=.13, t(234.08)=-1.82, p=.07, \beta=-.09,95 \% \mathrm{CI}_{\beta}[-.19, .01], \eta_{\mathrm{p}}{ }^{2}=.01$, nor was the three-way Target Status $\times$ Condition $\times$ Source interaction, $b=.09, S E=.06$, $t(234.99)=1.37, p=.17, \beta=.03,95 \% \mathrm{CI}_{\beta}[-.01, .08], \eta_{\mathrm{p}}{ }^{2}=.008$. This prompted no further consideration of those effects.

\section{Discussion}

We found continued evidence for how crowding shapes interpersonal preferences by considering putative cues to infectious disease. Crowd salience heightened preferences for conspecifics who appeared healthy over those who appeared diseased. However, this preference appears driven by reduced aversion to healthy conspecifics within a crowded ecology rather than enhanced aversion to diseased faces. Participants downregulated their aversion to healthy faces when crowding was salient, which could be in the service of ensuring closer proximity to those appearing most capable of satisfying disease avoidance goals (i.e., healthy; Brown, 2021; Brown \& Sacco, 2019; Young et al., 2011). Conversely, the signal value of disease cues could 
have been pervasive across conditions, resulting in both crowding-primed and control-primed participants similarly viewing the diseased face as aversive.

Given the overall aversion to both categories of faces within a crowded environment, these findings may explain why healthy faces were not rated as categorically favorable. It could be argued this preference exists as more of a tolerance toward these features, providing a degree of nuance for our preference prediction. The inevitability of interpersonal contact in crowds may motivate individuals to identify relatively safer conspecifics within these ecologies (see Maeng et al., 2013; Sng et al., 2017), which would ensure optimal fitness enhancement around those who would be less likely to induce high infection costs compared to those who appear diseased.

These findings may clarify inconsistencies in previous work by considering whether the features putatively connote infection risk. Although BIS responses typically operate through overgeneralizations of poor health as virulent (Haselton \& Nettle, 2006), crowding-induced responses may be specific to infection cues given the difficulty one may have with avoiding all disease vectors in a crowded environment. The preference for facial symmetry is rooted in perceptions of enhanced health, given its association with immunological functioning (e.g., Thornhill \& Gangestad, 2006; Young et al., 2011; cf. Pound et al., 2014). Conversely, previous work demonstrates crowd salience did not heighten aversion toward obesity (Brown \& Sacco, 2019), another physical cue inferred as diseased despite posing no infection risk (Miller \& Maner, 2012). The blemishes exhibited by targets in this study could have been inferred as diagnostic of infection risk, necessitating crowding-primed participants to upregulate tolerance of interpersonal contact with conspecifics who could pose less disease risk.

\section{Exploratory Regional Effects}

An exploratory component of our study indicated unexpected regional differences toward pathogenic cues. Southern participants reported greater aversion to faces possessing disease cues compared to Northern participants. This difference could reflect the former's awareness of higher environmental pathogen loads in the Southeastern U.S. compared to other regions (Feigenbaum, Muller, \& Wrigley-Field, 2019; Reif et al., 2017), which could manifest as previously reported reduced tolerance for nonnormative conspecifics across different cultures (Fincher \& Thornhill, 2012; Murray et al., 2011). Although aversion to disease was apparent for both regions, Southern participants' interpersonal wariness was larger, potentially reflecting an overgeneralization to potential infection risks (Haselton \& Nettle, 2006).

Crowd salience affected both regions' aversion similarly. This similarity could be rooted in a general ubiquity of the unique pathogenic threat imposed by crowding that fosters greater interpersonal restrictiveness regardless of one's region (Maeng et al., 2013; Sng et al., 2017). Individuals could similarly valuate crowds as threatening across cultures given the role of interpersonal contact in disease transmission (Jones et al., 2008).

Alternatively, despite being similar to findings from before 2020 (Brown \& Sacco, 2019), the fact that this study was conducted during the early stages of the 
COVID-19 pandemic could have heightened the salience of the interpersonal costs of extensive interpersonal contact unilaterally. It could have further been possible that the relatively heightened aversion to diseased faces at the Southern university could be partially explained by the fact that Northern states were already employing more extensive social distancing measures at this point in the pandemic than were Southern states (i.e., April and March 2020) to where immediate concerns of infection through disease cues could have elicited less aversion from participants in the Northern state. Nonetheless, it remains crucial to remember that the psychological responses to a pandemic may not necessarily be the same as the responses toward proximal disease cues to which humans evolved, given the historical recency of pandemics for the species (Ackerman, Tybur, \& Blackwell, 2021). Potential ambiguities from the pandemic necessitate future research to clarify regional effect, which could inform policies to leverage the interplay between affiliative and disease avoidance motives in shaping adherence to public health guidelines (Young, Brown, \& Sacco, 2021).

\section{Limitations and Future Directions}

Several limitations arise in this study that warrant future research. First, it is unclear how these findings translate into actual crowding experiences. Despite the current paradigm previously demonstrating considerable efficacy in activating the salience of existing within a crowd (e.g., Brown \& Sacco, 2019; Maeng et al., 2013), it may not address actual behaviors individuals may employ within crowds. Future research could consider placing participants in densely populated rooms before evaluating others or potentially have researchers identify the distance participants may stand from confederates displaying varying degrees of health (e.g., Aiello et al., 1977; Baum \& Valins, 1979). Confederates could additionally start coughing or sneezing while interacting with participants (Lee et al., 2010), which could further provide a more ancestrally relevant response to acute disease threat based on the presence of physical disgust cues (Kupfer \& Fessler, 2018).

It should be further noted that effects seeking to activate behavioral immune system responses through motivational priming have recently experienced mixed results, even those employing the same paradigm (e.g., Brown \& Sacco, 2016, 2019; Makhanova et al., 2019). Although the previous findings could reflect unforeseen nuance in understanding how disease primes could affect specific cognitions and perceptions (e.g., moral foundation endorsement is less malleable to situations, crowd salience is most robust in social perceptions), it remains necessary to identify the robustness of significant findings in the literature, especially considering smaller effect sizes in these studies. This concern makes direct replications, including the use of registered reports, necessary for many behavioral immune system studies.

Another potential limitation may concern the nature of the physical cues to disease displayed. Although connoting illness, these features are nonetheless approximations of actual disease cues. A future study could present images of targets actually infected with diseases and determine whether crowd salience heightens existing acuity to the infectious disease individuals possess, even without conspicuous sores 
(Axelsson et al., 2018; Tskhay, Wilson, \& Rule, 2016). Crowd salience should heighten preferences for faces not exhibiting disease cues. Though not putatively associated with infection risk, it could additionally be possible that heuristic associations between disease and foreign individuals could similarly heighten aversion to members of specific outgroups (e.g., Brown, Sacco, \& Medlin, 2019d; Moran et al., 2021; Navarrete \& Fessler, 2006). Future research could render crowding salient before tasking participants with evaluating targets from various ingroup or outgroup categories (i.e., foreign versus domestic individuals) while assessing the degree targets appear pathogenically threatening.

\section{Conclusion}

Population density in modern life is at odds with evolutionary history and serves to motivate various threat detection responses. One response includes identifying disease threats that pose infection risk through interpersonal contact. This work found additional evidence crowding heightens acuity toward disease cues to reduce infection risk in these environments.

Funding This research is supported by NSF Award 2030886.

\section{Declarations}

Conflict of interest On behalf of all authors, the first author reports no conflicts of interest.

\section{References}

Ackerman, J. M., Becker, D. V., Mortensen, C. R., Sasaki, T., Neuberg, S. L., \& Kenrick, D. T. (2009). A pox on the mind: Disjunction of attention and memory in the processing of physical disfigurement. Journal of Experimental Social Psychology, 45, 478-485.

Ackerman, J. M., Tybur, J. M., \& Blackwell, A. D. (2021). What role does pathogen-avoidance psychology play in pandemics? Trends in Cognitive Sciences, 25, 177-186.

Aiello, J. R., DeRisi, D. T., Epstein, Y. M., \& Karlin, R. A. (1977). Crowding and the role of interpersonal distance preference. Sociometry, 40(3), 271-282.

Ainsworth, S. E., \& Maner, J. K. (2019). Pathogen avoidance mechanisms affect women's preference for symmetrical male faces. Evolutionary Behavioral Sciences, 13, 265-271.

Al-Shawaf, L., Lewis, D. M., Alley, T. R., \& Buss, D. M. (2015). Mating strategy, disgust, and food neophobia. Appetite, 85, 30-35.

Axelsson, J., Sundelin, T., Olsson, M. J., Sorjonen, K., Axelsson, C., Lasselin, J., \& Lekander, M. (2018). Identification of acutely sick people and facial cues of sickness. Proceedings of the Royal Society B: Biological Sciences, 285, 20172430.

Baum, A., \& Valins, S. (1979). Architectural mediation of residential density and control: Crowding and the regulation of social contact. Advances in Experimental Social Psychology, 12, 131-175.

Bloom, D. E., Canning, D., \& Fink, G. (2008). Urbanization and the wealth of nations. Science, 319(5864), 772-775.

Brown, M. (2021). Goal relevance and desirability of virtuous behavior in satisfying affiliative and pathogen avoidance needs. Personality and Individual Differences, 181, 111025. 
Brown, M., Keefer, L. A., Sacco, D. F., \& Bermond, A. (2019a). Is the cure a wall? Behavioral immune system responses to a disease metaphor for immigration. Evolutionary Psychological Science, 5, 343-356.

Brown, M., \& Sacco, D. F. (2019). How and when crowd salience activates pathogen-avoidant motives. Evolutionary Behavioral Sciences. https://doi.org/10.1037/ebs0000191

Brown, M., \& Sacco, D. F. (2020). Testing the motivational tradeoffs between pathogen avoidance and status acquisition. Social Psychological Bulletin, 15, 1-22.

Brown, M., \& Sacco, D. F. (2016). Avoiding extraverts: Pathogen concern downregulates preferences for extraverted faces. Evolutionary Psychological Science, 2, 278-286.

Brown, M., Medlin, M. M., Sacco, D. F., \& Young, S. G. (2019b). Facing competing motives: Testing for motivational tradeoffs in affiliative and pathogen-avoidant motives via extraverted face preferences. Evolutionary Psychological Science, 5, 440-446.

Brown, M., Rodriguez, D. N., Gretak, A. P., \& Berry, M. A. (2017). Preliminary evidence for how the behavioral immune system predicts juror decision-making. Evolutionary Psychological Science, 3, 325-334.

Brown, M., Sacco, D. F., \& Medlin, M. M. (2019c). Approaching extraverts: Socially excluded men prefer extraverted faces. Personality and Individual Differences, 137, 198-203.

Brown, M., Sacco, D. F., \& Medlin, M. M. (2019d). Women's short-term mating goals elicit avoidance of faces whose eyes lack limbal rings. Evolutionary Behavioral Sciences, 13, 278-285.

Brown, M., Young, S. G., \& Sacco, D. F. (2021). Competing motives in a pandemic: Affiliative and pathogen-avoidance motives predict (non)compliance with social distancing guidelines. Computers in Human Behavior, 123, 106892.

Cain, C. K., \& LeDoux, J. E. (2008). Emotional processing and motivation: In search of brain mechanisms. In A. J. Elliot (Ed.), Handbook of approach and avoidance motivation (pp. 17-34). Psychology Press.

Chapuisat, M., Oppliger, A., Magliano, P., \& Christe, P. (2007). Wood ants use resin to protect themselves against pathogens. Proceedings of the Royal Society B: Biological Sciences, 274, 2013-2017.

Duncan, L. A., Schaller, M., \& Park, J. H. (2009). Perceived vulnerability to disease: Development and validation of a 15-item self-report instrument. Personality and Individual Differences, 47, 541-546.

Feigenbaum, J. J., Muller, C., \& Wrigley-Field, E. (2019). Regional and racial inequality in infectious disease mortality in US cities, 1900-1948. Demography, 56, 1371-1388.

Fincher, C. L., \& Thornhill, R. (2012). Parasite-stress promotes in-group assortative sociality: The cases of strong family ties and heightened religiosity. Behavioral and Brain Sciences, 35, 61-79.

Fincher, C. L., Thornhill, R., Murray, D. R., \& Schaller, M. (2008). Pathogen prevalence predicts human cross-cultural variability in individualism/collectivism. Proceedings of the Royal Society B: Biological Sciences, 275, 1279-1285.

Gagnon, L., Gagnon, S., \& Lloyd, J. (2020). Social distancing causally impacts the spread of SARSCoV-2: a US nationwide event study. Preprint available at MedRxiv.

Gochman, I. R., \& Keating, J. P. (1980). Misattributions to crowding: Blaming crowding for nondensitycaused events. Journal of Nonverbal Behavior, 4, 157-175.

Goodall, J. (1986). Social rejection, exclusion, and shunning among the Gombe chimpanzees. Ethology and Sociobiology, 7, 227-236.

Haselton, M. G., \& Nettle, D. (2006). The paranoid optimist: An integrative evolutionary model of cognitive biases. Personality and Social Psychology Review, 10, 47-66.

Hoang, T., Coletti, P., Melegaro, A., Wallinga, J., Grijalva, C. G., Edmunds, J. W., ... Hens, N. (2019). A systematic review of social contact surveys to inform transmission models of close-contact infections. Epidemiology, 30, 723-736.

Jones, K. E., Patel, N. G., Levy, M. A., Storeygard, A., Balk, D., Gittleman, J. L., \& Daszak, P. (2008). Global trends in emerging infectious diseases. Nature, 451, 990-993.

Judd, C. M., McClelland, G. H., \& Ryan, C. S. (2017). Data analysis: A model comparison approach to regression, ANOVA, and beyond. Routledge.

Kavaliers, M., Choleris, E., \& Pfaff, D. W. (2005). Genes, odours and the recognition of parasitized individuals by rodents. Trends in Parasitology, 21, 423-429.

Kennedy, D. P., Gläscher, J., Tyszka, J. M., \& Adolphs, R. (2009). Personal space regulation by the human amygdala. Nature Neuroscience, 12, 1226-1227.

Kluger, M. J. (1991). Fever: Role of pyrogens and cryogens. Physiological Reviews, 71, 93-127. 
Kupfer, T. R., \& Fessler, D. M. (2018). Ectoparasite defence in humans: Relationships to pathogen avoidance and clinical implications. Philosophical Transactions of the Royal Society B: Biological Sciences, 373, 20170207.

Lee, S. W., Schwarz, N., Taubman, D., \& Hou, M. (2010). Sneezing in times of a flu pandemic: Public sneezing increases perception of unrelated risks and shifts preferences for federal spending. Psychological Science, 21, 375-377.

Litman, G. W., Cannon, J. P., \& Dishaw, L. J. (2005). Reconstructing immune phylogeny: New perspectives. Nature Reviews Immunology, 5, 866-879.

Maeng, A., Tanner, R. J., \& Soman, D. (2013). Conservative when crowded: Social crowding and consumer choice. Journal of Marketing Research, 50, 739-752.

Makhanova, A., Plant, E. A., \& Maner, J. K. (2021). Capturing fluctuations in pathogen avoidance: The situational pathogen avoidance scale. Evolutionary Psychological Science, 7, 21-38.

Makhanova, A., Plant, E. A., Monroe, A. E., \& Maner, J. K. (2019). Binding together to avoid illness: Pathogen avoidance and moral worldviews. Evolutionary Behavioral Sciences, 13, 182-204.

Makhanova, A., \& Shepherd, M. A. (2020). Behavioral immune system linked to responses to the threat of COVID-19. Personality and Individual Differences, 167, 110221.

Mortensen, C. R., Becker, D. V., Ackerman, J. M., Neuberg, S. L., \& Kenrick, D. T. (2010). Infection breeds reticence: The effects of disease salience on self-perceptions of personality and behavioral avoidance tendencies. Psychological Science, 21, 440-447.

Miller, S. L., \& Maner, J. K. (2012). Overperceiving disease cues: The basic cognition of the behavioral immune system. Journal of Personality and Social Psychology, 102, 1198-1213.

Murray, D. R., \& Schaller, M. (2012). Threat(s) and conformity deconstructed: Perceived threat of infectious disease and its implications for conformist attitudes and behavior. European Journal of Social Psychology, 42, 180-188.

Murray, D. R., \& Schaller, M. (2016). The behavioral immune system: Implications for social cognition, social interaction, and social influence. Advances in Experimental Social Psychology, 53, 75-129.

Murray, D. R., Schaller, M., \& Suedfeld, P. (2013). Pathogens and politics: Further evidence that parasite prevalence predicts authoritarianism. PLOS ONE, 8, e62275.

Murray, D. R., Trudeau, R., \& Schaller, M. (2011). On the origins of cultural differences in conformity: Four tests of the pathogen prevalence hypothesis. Personality and Social Psychology Bulletin, 37, 318-329.

Moran, J. B., Goh, J. X., Kerry, N., \& Murray, D. R. (2021). Outbreaks and outgroups: Three tests of the relationship between disease avoidance motives and xenophobia during an emerging pandemic. Evolutionary Psychological Science. https://doi.org/10.1007/s40806-021-00283-z

Navarrete, C. D., \& Fessler, D. M. (2006). Disease avoidance and ethnocentrism: The effects of disease vulnerability and disgust sensitivity on intergroup attitudes. Evolution and Human Behavior, 27, 270-282.

Neuberg, S. L., Kenrick, D. T., \& Schaller, M. (2011). Human threat management systems: Self-protection and disease avoidance. Neuroscience and Biobehavioral Reviews, 35, 1042-1051.

Pancer, Z., \& Cooper, M. D. (2006). The evolution of adaptive immunity. Annual Review of Immunology, 24, 497-518.

Peng, M., Chang, L., \& Zhou, R. (2013). Physiological and behavioral responses to strangers compared to friends as a source of disgust. Evolution and Human Behavior, 34, 94-98.

Pound, N., Lawson, D. W., Toma, A. M., Richmond, S., Zhurov, A. I., \& Penton-Voak, I. S. (2014). Facial fluctuating asymmetry is not associated with childhood ill-health in a large British cohort study. Proceedings of the Royal Society B: Biological Sciences, 281, 20141639.

Reif, S., Safley, D., McAllaster, C., Wilson, E., \& Whetten, K. (2017). State of HIV in the US Deep South. Journal of Community Health, 42, 844-853.

Rocklöv, J., \& Sjödin, H. (2020). High population densities catalyse the spread of COVID-19. Journal of Travel Medicine, 27, taaa038.

Rotella, A., Varnum, M. E., Sng, O., \& Grossmann, I. (2020). Increasing population densities predict decreasing fertility rates over time: A 174-nation investigation. American Psychologist. https://doi. org/10.31234/osf.io/zpc7t

Sacco, D. F., Young, S. G., \& Hugenberg, K. (2014). Balancing competing motives: Adaptive trade-offs are necessary to satisfy disease avoidance and interpersonal affiliation goals. Personality and Social Psychology Bulletin, 40, 1611-1623. 
Salathé, M., Kazandjieva, M., Lee, J. W., Levis, P., Feldman, M. W., \& Jones, J. H. (2010). A highresolution human contact network for infectious disease transmission. Proceedings of the National Academy of Sciences of USA, 107, 22020-22025.

Sawada, N., Auger, E., \& Lydon, J. E. (2018). Activation of the behavioral immune system: Putting the brakes on affiliation. Personality and Social Psychology Bulletin, 44, 224-237.

Schaeffer, G. H., \& Patterson, M. L. (1980). Intimacy, arousal, and small group crowding. Journal of Personality and Social Psychology, 38, 283-290.

Schaller, M., \& Murray, D. R. (2008). Pathogens, personality, and culture: Disease prevalence predicts worldwide variability in sociosexuality, extraversion, and openness to experience. Journal of Personality and Social Psychology, 95, 212-221.

Sng, O., Neuberg, S. L., Varnum, M. E. W., \& Kenrick, D. T. (2017). The crowded life is a slow life: Population density and life history strategy. Journal of Personality and Social Psychology, 112, 736-754.

Stockdale, J. E. (1978). Crowding: Determinants and effects. Advances in Experimental Social Psychology, 11, 197-247.

Thornhill, R., \& Gangestad, S. W. (2006). Facial sexual dimorphism, developmental stability, and susceptibility to disease in men and women. Evolution and Human Behavior, 27, 131-144.

Tybur, J. M., Lieberman, D., Fan, L., Kupfer, T. R., \& de Vries, R. E. (2020). Behavioral immune tradeoffs: Interpersonal value relaxes social pathogen avoidance. Psychological Science, 31, 1211-1221.

Tybur, J. M., \& Lieberman, D. (2016). Human pathogen avoidance adaptations. Current Opinion in Psychology, 7, 6-11.

Tskhay, K. O., Wilson, J. P., \& Rule, N. O. (2016). People use psychological cues to detect physical disease from faces. Personality and Social Psychology Bulletin, 42, 1309-1320.

van Leeuwen, F., \& Petersen, M. B. (2018). The behavioral immune system is designed to avoid infected individuals, not outgroups. Evolution and Human Behavior, 39, 226-234.

Wang, I. M., \& Ackerman, J. M. (2019). The infectiousness of crowds: Crowding experiences are amplified by pathogen threats. Personality and Social Psychology Bulletin, 45, 120-132.

Whitaker, S. D. (2021). Did the COVID-19 pandemic cause an urban exodus? Cleveland Fed District Data Briefs (pp. 1-46).

Wu, B. P., \& Chang, L. (2012). The social impact of pathogen threat: How disease salience influences conformity. Personality and Individual Differences, 53, 50-54.

Young, S. G., Brown, M., \& Sacco, D. F. (2021). Using psychological science to support social distancing: Tradeoffs between affiliation and disease-avoidance motivations. Social and Personality Psychology Compass, 15, e12597.

Young, S. G., Sacco, D. F., \& Hugenberg, K. (2011). Vulnerability to disease is associated with a domainspecific preference for symmetrical faces relative to symmetrical non-face stimuli. European Journal of Social Psychology, 41, 558-563.

Zebrowitz, L. A., \& Rhodes, G. (2004). Sensitivity to "bad genes" and the anomalous face overgeneralization effect: Cue validity, cue utilization, and accuracy in judging intelligence and health. Journal of Nonverbal Behavior, 28, 167-185.

Publisher's Note Springer Nature remains neutral with regard to jurisdictional claims in published maps and institutional affiliations. 